\section{Localized Hypertrichosis after Index Finger Revascularization and Complex Regional Pain Syndrome}

Gottfried Wechselberger ${ }^{1}$, Karl Schwaiger ${ }^{2}$, Dolores Wolfram ${ }^{3}$

${ }^{1}$ Deparment of Plastic, Aesthetic and Reconstructive Surgery, Hospital of Barmherzige Brüder Salzburg, Salzburg; ${ }^{2}$ Paracelsus Medical University Salzburg, Salzburg; ${ }^{3}$ Department of Plastic, Aesthetic and Reconstructive Surgery, University Hospital of Innsbruck, Innsbruck, Austria

Correspondence: Gottfried Wechselberger

Department of Plastic, Aesthetic and Reconstructive Surgery, Hospital of the Barmherzige Brüder (St. John of God) Salzburg, Academic Teaching Hospital of the Paracelsus Medical University, 5010 Salzburg, Austria

Tel: +436628088-8452, Fax: +436628088-8452

E-mail: gottfried.wechselberger@bbsalz.at

No potential conflict of interest relevant to this article was reported.

Received: 15 Mar 2014 • Revised: 15 Apr 2014 • Accepted: 15 Apr 2014 pISSN: 2234-6163・ elSSN: 2234-6171

http://dx.doi.org/10.5999/aps.2014.41.5.603 • Arch Plast Surg 2014;41:603-604

Copyright (C) 2014 The Korean Society of Plastic and Reconstructive Surgeons This is an Open Access article distributed under the terms of the Creative Commons Attribution Non-Commercial License (http://creativecommons.org/licenses/by-nc/3.0/) which permits unrestricted non-commercial use, distribution, and reproduction in any medium, provided the original work is properly cited.

Various skin changes have been described in complex regional pain syndrome (CRPS), but the association of hypertrichosis with finger replantation has not been described in literature. CRPS occurs as the inadequate response to painful trauma in a distal extremity. Clinically, two types of CRPS can be differentiated according to the preceding noxious event without (CRPS I) or with (CRPS II) an obvious nerve lesion [1]. CRPS I (sympathetic reflex dystrophy) is characterized by sensory disturbances, burning deep spontaneous pain, and allodynia. Disturbances in the skin blood circulation, sweating, edema, and trophic disturbances of the skin, joints, and bones are typical and not limited to the region of the peripheral nerves. CRPS II (causalgia) appears following a partial peripheral nerve lesion, but the symptoms are identical [1]. Regarding the pathogenetic mechanism involved in CRPS, no consensus exists, but traumarelated cytokine release, neurogenic inflammation, sympathetically maintained pain, and cortical reorganization in response to chronic pain seem to play a key role in the development and maintenance of this complex pain syndrome [2].

We describe a patient who developed a mild form of CRPS with an evident localized hypertrichosis on the hand and forearm after subtotal amputation and revascularization of the distal phalanx of the index finger.

A 26-year-old woman presented with a traumatic subtotal amputation of the distal phalanx of the right index finger caused by a woodworking machine. Under general anesthesia, two crossed Kirschner wires were used for bony fixation, and for revascularization, the proper digital artery supplying the ulnar side and two dorsal veins were repaired. Both digital nerves were intact. The immediate postoperative course was uneventful, but on the third postoperative day, a thrombosis of the reconstructed artery occurred, which required surgical revision. Under general anesthesia and continuous brachial plexus blockade, reconstruction and revascularization were performed by interposition of a vein graft from the forearm. Continuous brachial plexus blockade for increased blood flow to the injured extremity and postoperative pain management were retained for 5 days. Postoperatively, the index finger was dressed with gauze and a simple hand bandage. Occupational therapy was started 2 weeks postoperatively.

About 5 weeks after surgery, the patient presented
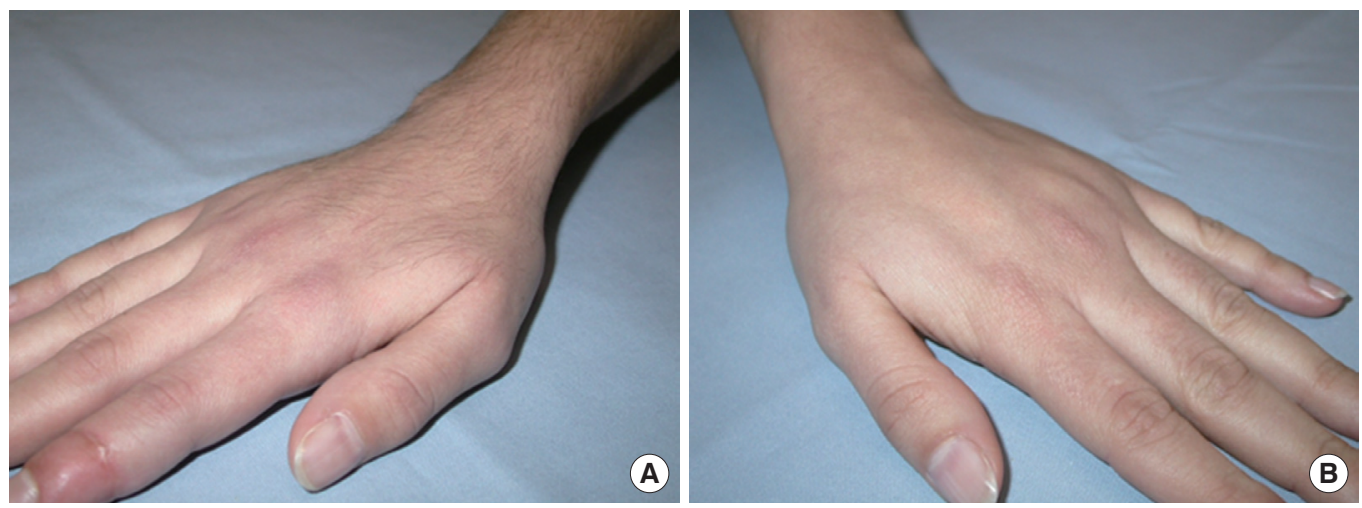

Fig. 1.

(A) Right hand with local hypertrichosis. (B) Left hand. 
with a swelling and a glassy dermal texture of the index finger and the hand with expansion to the forearm. Active range of motion was reduced, but the patient did not complain about severe pain. Occupational therapy was continued and calcitonin, in the form of a nasal spray, was prescribed. Six weeks later, a distinct local hypertrichosis of the right hand and forearm was observed, even though at this time, edema and functional impairment had nearly disappeared (Fig. 1). Six months after surgery, the patient was free of complaints, and the localized hypertrichosis was in involution.

CRPS is a complex syndrome that is most often initiated by trauma to an extremity, such as fractures, surgical wounds, and crush injuries. It is rarely attributed to other injuries such as venipuncture, burns, electrical injuries, inflammatory processes, and spinal cord injuries. Unusual precipitating events supposedly account for CRPS, including central nervous system lesions, peripheral vascular bypass procedures, and carpal tunnel syndrome. The exact pathomechanism of this complex syndrome is not clarified, but the major mechanisms, which might occur subsequently or in parallel during the course of CRPS, are trauma-related cytokine release, dysfunction of thermoregulation, neurogenic inflammation, sympathetically maintained pain, and cortical changes in response to pain [2]. Further, molecular biological examination has pointed to an association with human leukocyte antigen (HLA), and the opinion that CRPS has a psychosomatic background is also popular [2].

Although in our case, the chronic pain was not ostensible, four of the five symptoms (unexplained diffuse pain, altered skin color, altered skin temperature, edema, and reduced active range of motion) were present to justify the diagnosis of CRPS.

Unilateral functional inhibition of sympathetic vasoconstrictor neurons seems to be a characteristic feature of acute CRPS and is unlikely to be responsible for the skin warming and vasodilatation. Therefore, continuous brachial plexus blockade for increased blood flow to the injured extremity and postoperative pain management could have been a trigger in the development of CRPS in our patient.

Regarding the therapy of this complex syndrome, efficient pain therapy (non-steroidal antiinflammatory drugs and opioids) and pharmacologic medical treatment (sympatholytics, anticonvulsants, corticoids, antidepressants, anxiety medications, and calcitonin), aside from occupational therapy, are mainly responsible for a good clinical outcome [1]. In case of deficient improvement of disorders, an invasive treatment (stellate ganglion and other nerve blocks) can be discussed. However, in our case, the conservative therapy concept with cooling and keeping the affected finger in an elevated position as often as possible, as well as the intensive occupational therapy (active and passive extension/flexion exercises of the whole hand), were sufficient to disrupt the vicious circle of CRPS and to obtain good hand function. Whether intranasal calcitonin application has a positive effect, is controversial in the literature [3].

Localized hypertrichosis has been reported to develop at the site of malignancies, psoralen+ ultraviolet-A light therapy, and infection (chickenpox scars); at the periphery of burned skin; in the leg after radical inguinal lymphadenectomy; and after topical use of diverse medicaments (such as minoxidil, prostaglandine, and glucocorticoids) and is also reported in connection with CRPS [4]. Pathogenically, we postulate that the cutaneous hyperemia, as a part of the CRPS, induced the hypertrichosis by stimulating the hair follicles [5]. Further, the anxious personality of our patient, the fear of overloading the transplanted finger, and the subsequent prolonged immobilization, could have played a key role in the manifestation of the symptoms.

\section{References}

1. Baron R, Binder A, Ulrich W, et al. Complex regional pain syndrome. Sympathetic reflex dystrophy and causalgia. Schmerz 2003;17:213-26.

2. Birklein F. Complex regional pain syndrome. J Neurol 2005;252:131-8.

3. Arlet J, Mazieres B. Medical treatment of reflex sympathetic dystrophy. Hand Clin 1997;13:477-83.

4. Kandi B, Kaya A, Turgut D, et al. Clinical presentation of cutaneous manifestations in complex regional pain syndrome (type 1). Skinmed 2007;6:118-21.

5. Montagna W, Ellis RA. The vascularity and innervation of human follicles. In: Montagna W, Ellis RA, editors. The biology of hair growth. New York: Academic Press; 1958. p.232-40. 